\title{
UN TEXTO DESCONOCIDO DEL BOTÁNICO BALTASAR BOLDO
}

\author{
Fco. Javier Dosil Mancilla
}

BALTASAR BOLDO, La producción marítima particular observada en La Coruña, Santiago, Ignacio Aguayo, 1797.

En el presente trabajo se reseña un folleto del médico y naturalista aragonés Baltasar Boldo que, por su escasa difusión, parece haber pasado inadvertido hasta el presente, a pesar de que aporta datos de cierto interés sobre su vida y su contribución a la ciencia. El trabajo fue localizado entre los folletos del naturalista coruñés José Cornide Saavedra (1734-1803), en el Archivo del Reino de Galicia (La Coruña) ${ }^{1}$.

El nombre de Baltasar Manuel Boldo Tuced figura en general ligado a la Real Comisión de Guantánamo (1796-1802), viaje exploratorio a Cuba proyectado por el Conde de Mopox con el principal objetivo de trazar un canal desde los montes de Güinas, al oeste de la isla, hasta el puerto de La Habana, para facilitar el transporte de la madera, requerida en grandes cantidades para la fabricación de buques de guerra ${ }^{2}$. Por recomendación del intendente del Real Jardín Botánico, recayó en Baltasar Boldo la tarea de examinar las diversas clases de madera y estudiar las plantas recolectadas en la expedición, si bien, por propio interés del naturalista, se ampliaría su cometido al estudio de otras producciones natura-

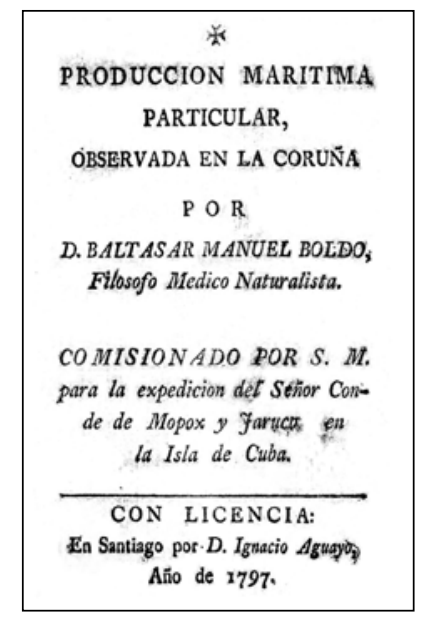

1 Archivo del Reino de Galicia, Fondo: José Cornide Saavedra, leg. 11, nº 200, La Coruña.

2 Puede consultarse una relación de la bibliografía sobre la Comisión de Guantánamo en A. Gomis Blanco, F. Pelayo López \& J. Fernández Pérez, «Valoración de los resultados obtenidos por los naturalistas de la expedición a Cuba del Conde de Mopox (1796-1802)», en Estudios sobre Historia de la Ciencia $y$ de la Técnica, Valladolid, Junta de Castilla León, 1988, pp. 631-638. A esta lista pueden añadirse otros trabajos más recientes: A. Gomis Blanco, «Las Ciencias Naturales en la expedición del Conde de Mopox a Cuba», en A.R. Díez Torres et al. (coords.) La Ciencia Española en Ultramar, Madrid, Ateneo de Madrid-Doce Calles, 1991, pp. 309-319; A. Gomis Blanco \& F. Pelayo López, «Baltasar M. Boldo y la Real Comisión de Guantánamo del Conde de Mopox», en M. P. de San Pío Aladrén \& M. A. PuigSamper Mulero (coords.) Las Flores del Paraíso. La expedición botánica de Cuba en los siglos XVIII y XIX, Barcelona, Lunwerg, 1999, pp. 75-92, y M.A. Puig-Samper \& L. Maldonado, «La botánica en la Real Comisión de Guantánamo (1796-1802)», en J. Fernández Casas, M.A. Puig-Samper \& F.J. Sánchez García (eds.), «Cubensis prima flora seu descripciones diversorum generum specierumque insulae Cubae plantarum quas Regia Guantanamensis Legatio inspexit ...», Fontequeria, 29, 1990, pp. 5-17. 
les $^{3}$. A pesar del protagonismo desempeñado por Boldo en esta Comisión, que lo ha hecho merecedor de diversos estudios biográficos, son muchas las lagunas que se mantienen en torno a su vida y su actividad científica ${ }^{4}$.

Nació en Zaragoza, probablemente en $1766^{5}$. En esta ciudad realizó sus primeros estudios, de Teología, y en ella alcanzó el grado de Bachiller en Medicina, tras cuatro años de formación en la Universidad de Valencia. Más tarde revalidó el título en Madrid y obtuvo por oposición la plaza de Médico de Entradas en los Reales Hospitales General y de la Pasión de Madrid. Ejerció la Medicina en Zaragoza, obteniendo brillantes resultados, hasta 1793, año en que fue nombrado médico de número de los Reales Ejércitos con destino en el Hospital de Palamós. Hizo tres campañas (hasta 1795), prestando importantes servicios que le valieron el título de Protomédico de Rosellón y Consultor Perpetuo. Su curiosidad científica le llevó además a realizar diversos trabajos químicos, botánicos y epidemiológicos en Cataluña y Baleares, que determinaron su ingreso, como Miembro Correspondiente, en el Real Jardín Botánico de Madrid y en la Academia de Buenas Letras de Barcelona.

En 1796 fue elegido para observar las virtudes de las plantas cultivadas en el Jardín Botánico madrileño, tarea que simultaneó con la atención médica del personal del centro. Sin embargo, se dedicó poco tiempo a estas actividades, ya que en el mismo año, el Gobierno lo incorporó como primer botánico a la mencionada comisión científica a Cuba. La expedición partió del puerto de La Coruña el 3 de diciembre de 1796, a bordo de la fragata El Rey mandada por Pedro Núñez y en la que viajaban, además del propio conde de Mopox, los naturalistas José Guío, Francisco Remírez y Félix Bourman, diversos ingenieros y varios empleados militares 6 . Llegó a Cuba en febrero de 1797, tras una escala de varios días en Santa Cruz de Tenerife, forzada por los fuertes temporales. En los dos años siguientes - falleció en La Habana, de forma inesperada, el 31 de julio de 1799recolectó plantas y animales, exploró la parte occidental de la isla acompañado por Martín de Sessé, realizó un viaje de carácter científico a Estados Unidos y redactó parte de una obra sobre la flora cubana, que permaneció en forma de manuscrito hasta hace unos años ${ }^{7}$.

Esta breve introducción nos ayudará a comprender las circunstancias en que fue elaborado el folleto de veinte páginas que reseñamos, titulado «Producción marítima particular, observada en La Coruña», y que fue publicado en 1797, en la imprenta de Ignacio Aguayo, en Santiago de Compostela. La producción marítima a la que se refiere el título no es sino una cápsula cerrada de un huevo de raya, que el naturalista recolectó en la bahía del Orzán, en la ciudad de La Coruña, después de «los fuertes Noroestes, que reynaron en esta Ciudad en el 16, 17 y 18 de este mes de Noviembre»,

3 Gomis Blanco \& Pelayo López, op. cit., p. 79.

4 Los datos biográficos de Boldo pueden consultarse en M. Colmeiro, La botánica y los botánicos de la Península Hispano-Lusitana. Estudios bibliográficos y biográficos, Madrid, Imp. M. Rivadeneyra, 1858, pp. 186-187, y especialmente en los diversos estudios de V. Martínez Tejero: Botánica aragonesa. IV Muestra de Documentación Histórica Aragonesa, Zaragoza, Diputación General de Aragón, 1991, pp. 41-43; «La ilustración aragonesa en América: Naturalistas en Cuba», Rolde, 52-53, 1990, pp. 4-15; Los botánicos aragoneses, Zaragoza, Caja de Ahorros de la Inmaculada de Aragón, 1998, pp. 49-51, y «La Botánica en Aragón hasta el siglo XIX», en Congreso de Botánica en homenaje a Francisco Loscos (1823-1886), Teruel, Instituto de Estudios Turolenses, 2000, pp. 13-43.

5 Francisco Guerra, en su Bibliografía médica americana y filipina, Madrid, Ollero \& Ramos, tomo 2,1999 , p. 323, señala 1746 como su fecha de nacimiento pero no indica las fuentes consultadas.

6 Gomis Blanco \& Pelayo López, op. cit., p. 80.

7 El manuscrito lleva el título Descripciones de diferentes géneros y especies de plantas de la isla de Cuba que ha examinado la Comisión Real de Guantánamo. En 1984 se publicó en Madrid, Turner, la parte iconográfica, con el título Flora y fauna cubanas del siglo XVIII: los dibujos de la expedición del conde de Mopox, 1796-1802. 
que «alteraron la mar, de tal suerte que las olas como montañas venian á estrellarse con grande estruendo en las rocas de las orillas, subiendo las aguas á alturas grandisimas» [p. 12]. Como consecuencia de este intenso temporal «murieron muchos pescados estrellados contra las peñas, y la resaca traxo á la orilla infinidad de Fucus, algas, Coralinas, Conservas, conchas, y otras producciones habiendo salido entre ellas un huevo de los que queda» [p. 12-13]. Estos detalles y su fecha de publicación nos hacen suponer que el trabajo fue redactado en La Coruña, a finales de noviembre o en los dos primeros días de diciembre de 1796, es decir, en los días previos a su partida a Cuba en el seno de la referida comisión. Este hecho se confirma con la explicación de la portada, en donde el autor figura como «Filosofo Medico Naturalista. Comisionado por S.M. para la expedicion del Señor Conde de Mopox y Jaruco, en la Isla de Cuba». Se trata, por lo tanto, de la primera contribución llevada a cabo por la Real Comisión de Guantánamo, y una de las pocas publicadas, ya que a pesar de los interesantes hallazgos, que fueron la base de numerosas láminas de dibujos de plantas y animales, los resultados de la expedición permanecieron en general inéditos ${ }^{8}$.

En el estudio que presentamos, Boldo se propone aclarar la auténtica naturaleza del objeto descubierto entre las algas de los arribazones de La Coruña. Advierte que no se trata de una producción extraña; al contrario, «es bastante común en las Playas que circundan la España por la parte del Mediodía en las Islas del Mediterráneo, en las Costas Norte, y señaladamente en el seno del Orzán, uno de los mas inmediatos à esta famosa Capital de Galicia» (pp. 1-2), a pesar de lo cual, todavía existía una gran confusión en torno a su auténtica naturaleza, que había suscitado las más diversas interpretaciones por parte de los naturalistas, como reflejaban los nombres con los que era conocida: «Hablo de una producción llamada en las costas españolas del Mediterráneo Escarabajo marítimo, en las del oceano Murciélago de mar, y entre algunos naturalistas Fucus vegigoso» (pp. 2-3). Para resolver la incógnita de su naturaleza, realiza, en primer lugar, una descripción morfológica de un ejemplar, en la que sólo se echa en falta referencias al tamaño de la cápsula, que le lleva a

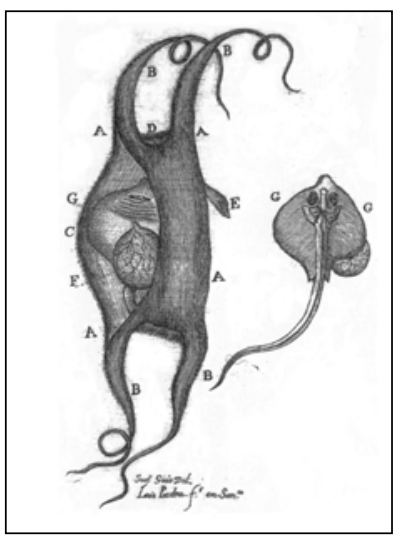
señalar lo inapropiado de estos nombres populares, pues saltaba a la vista que no se trataba ni de un insecto ni de un murciélago: «Mayor probabilidad tuvieron los que lo reputaron por un Fucus vegigoso. Lo primero, porque hay algunos de estos que (aunque menores) tienen vegigas semejantes à las de esta producción. En esta costa he encontrado hasta nueve especies de Fucus vegigosos muy raros. Lo segundo, porque esta produccion parece formada de una materia elastica, y las vegigas de los referidos Fucus lo son igualmente. Lo tercero, porque los bordones, ó filamentos de que habemos hablado se parecen algo à varios Fucus filamentosos» (pp. 7-8); pero observa que tampoco comparte ciertas características importantes con los vegetales. "Infierese de aquí, que todas las opiniones expuestas tienen mui poca probabilidad, y que por consiguiente son inadmisibles, debiendo nosotros buscar la verdadera, apoyada en la historia exacta de esta producción» (p. 9).

A continuación, Boldo se propone demostrar definitivamente que se trata de un huevo de pez - como ya habían defendido algunos naturalistas, entre otros el mallorquín Cristóbal Vilella-, aprovechando la oportunidad que le ofrece el hallazgo, entre las algas de la bahía coruñesa, de un

8 Martínez Tejero, «La botánica en Aragón hasta el siglo XIX», p. 23, señala la publicación en La Habana, en 1798, de una epístola latina de Boldo dirigida al botánico y médico valenciano Tomás Villanova, con el título Bot. Reg. Caesaraug. in insulam cubensem nunc legatus D.O. Thomae Villanova, Bot. Prof. Valent. S.P.D. Havanae. 
ejemplar completo y bien conservado, que por encontrarse cerrado le hace suponer que «tal vez fuese recientemente puesto, y que á pesar de sus defensas los fuertes embates de la mar lo huviesen arrojado á la orilla antes de verificarse la fecundación, y salida del fetus» (p. 13). Así pues, se formula una serie de preguntas, que esperaba poder dar respuesta tras la observación de su interior, mediante una disección minuciosa: «¿Este grande huevo era acaso un receptáculo de otros infinitos huevecitos, como sucede en los de las truchas, barbos, tencas, sabogas, y madrillas en los rios; en los del atun, salmon, besugo, sardina, \& c. en los mares, ó contenia solo un fetus, sirviendole de amion? ¿De que pescado era este huevo? ¿Por qué agentes se actuaba su fecundación?» (pp. 1112). «Para salir de mis dudas resolví hacer anatomia de él: abrilo con mucho tiento por la costura de un costado, y observé los siguiente» (p. 14). Lo que observó en su interior fue «un huevo mui hermoso de la magnitud de una nuez pequeña», que se unía por un corto ligamento a «una carnosidad blanquecina pegada á una de las paredes interiores del referido saco [...]. Pareciome al principio ser esta la placenta de aquel huevo: pensé despues si haria las veces de un ligamento compresivo para sujetar al huevo, y impidiendole que en los movimientos del mar diese frecuentes choques contra las paredes de la bolsa en que estaba contenido. Pero, examinandole con mas escrúpulo, hallé ser un fetus ya fecundado y que en la formación de la cabeza cuerpo, alas y cola manifestaba ser el pescado conocido con el nombre de Raya» (pp. 15-16). Así pues, estas observaciones confirmaban la opinión de otros naturalistas, acerca de la función reproductiva de la producción marítima, e iban más allá al identificar el pez responsable con la raya, al reconocer el embrión y la vesícula vitelina, $\mathrm{y}$ al señalar que «en rigor no es el huevo sino el utriculo ovario amion, ò sea saco membranoso que le contiene» (p. 19), es decir, la cápsula u ooteca.

A continuación, el naturalista se plantea una serie de incógnitas que quedaban todavía sin resolver (cuál es el agente que produce la fecundación, cuántos huevos se producen en cada fecundación y qué peces se propagan por este mismo medio) y, en una nota que cierra el estudio, transfiere la continuación de estas investigaciones al físico y médico del Consulado de La Coruña, Francisco Cónsul Jove, «quien ha presenciado todas estas observaciones, y que no solo queda con el cuidado de la publicación y remesa de esta pieza curiosa, sino tambien de averiguar por medio de la diseccion el estado y numero en que se hallan estas producciones en los vientres de las Rayas en las diversas estaciones del año» (p. 20). Que fue Francisco Cónsul el responsable de la publicación del folleto, parece probado por el hecho de que su impresor no fuera otro que Ignacio Aguayo, de Santiago de Compostela, a quien el propio Cónsul había confiado, unos años antes, la publicación de algunos de sus trabajos ${ }^{9}$. En lo que se refiere a la continuación de las investigaciones, no nos consta que se haya producido, ni sabemos si llegó a remitir el ejemplar al Gabinete de Historia Natural de Madrid, previamente «infundido en espiritu de vino» (p. 17) por el propio Boldo. El estudio va acompañado de una lámina con un dibujo de un ejemplar diseccionado y un detalle del embrión, realizado por el madrileño José Guío Sánchez, quien también formaba parte de la Real Comisión de Guantánamo, en calidad de dibujante. Con anterioridad, Guío había participado como disector y dibujante principal de la flora y fauna en la famosa expedición científica de Alejandro Malaspina (1789-1794), bajo la dirección del botánico Luis Née10.

9 Efectivamente, figuran al menos dos obras de Cónsul publicadas en los talleres de Ignacio Aguayo: Memoria sobre el conocimiento de las tierras ... (1786), y Ensayo sobre hidráulica rústica ... (1788). Más detalles sobre el impresor Ignacio Aguayo en M. Soto Freire, La imprenta en Galicia. Ensayo bibliográfico, Santiago de Compostela, Xunta de Galicia, 1998, pp. 182-187.

10 Cf. C. Sotos Serrano, «La botánica y el dibujo en el siglo XVIII», en A.A.V.V., La botánica en la expedición Malaspina, 1789-1794, Madrid, Turner, 1989, pp. 71-79, y J. Torres Revello, Los artistas pintores de la Expedición Malaspina, Buenos Aires, Instituto de Investigaciones Históricas, vol. 2, 1944, pp. 4-77. 
Aunque de forma indirecta, el folleto aporta algunos datos sobre las relaciones científicas de Boldo. Ya se ha comentado el estrecho contacto que estableció en La Coruña con Francisco Cónsul Jove i Tineo (1754-1810), médico y físico asturiano, por entonces asociado al Real Consulado Marítimo y Terrestre de La Coruña, ${ }^{11}$. Probablemente lo conoció en el mismo Consulado, institución creada once años antes con el objeto de revitalizar el comercio con América, y cuya visita probablemente resultaba obligada para los expedicionarios que partían del puerto coruñés. Sabemos también que en sus exploraciones anteriores realizadas por las islas Baleares, estableció contactos científicos con el pintor y naturalista mallorquín Cristóbal Vilella (1742-1803) ${ }^{\mathbf{1 2}}$, quien puso a su disposición sus colecciones de Historia Natural.

11 Díaz-Fierros Viqueira, «Estudio preliminar», en F. Cónsul Jove, Memoria sobre el conocimiento de las tierras; verdadero i economico metodo de cultivarlas, adaptado al clima, i circunstancias de Galicia, i Asturias (ed. facsímil), Sada, O Castro, 1987, pp. 9-19, ofrece una síntesis biográfica de Francisco Cónsul.

12 La biografía de Cristóbal Vilella puede consultarse en I. Azcárate, Naturaleza y arte: la fauna de la isla de Mallorca en la obra de Cristóbal Vilella, Palma de Mallorca, José de Olañeta, 1990, pp. 61-89. 
